\title{
Anisotropies in magnetic field evolution and local Lyapunov exponents
}

\author{
X. Z. Tang* \\ Princeton Plasma Physics Laboratory, Princeton University, Princeton, NJ 08543
}

A. H. Boozer

Department of Applied Physics and Applied Mathematics, Columbia University, New York, NY 10027

(September 7, 1999)

*Email: xtang@pppl.gov 


\begin{abstract}
The natural occurrence of small scale structures and the extreme anisotropy in the evolution of a magnetic field embedded in a conducting flow is interpreted in terms of the properties of the local Lyapunov exponents along the various local characteristic (un)stable directions for the Lagrangian flow trajectories. The local Lyapunov exponents and the characteristic directions are functions of Lagrangian coordinates and time, which are completely determined once the flow field is specified. The characteristic directions that are associated with the spatial anisotropy of the problem, are prescribed in both Lagrangian and Eulerian frames. Coordinate transformation techniques are employed to relate the spatial distributions of the magnetic field, the induced current density, and the Lorentz force, which are usually followed in Eulerian frame, to those of the local Lyapunov exponents, which are naturally defined in Lagrangian coordinates.
\end{abstract}

PACS numbers: $05.60 .+\mathrm{w}, 05.45 .+\mathrm{b}, 52.55$.Dy, 47.10.+g

Typeset using REVTEX 


\section{INTRODUCTION}

Under the usual assumptions that the displacement current is negligible and the plasma obeys the generalized Ohm's law $\mathbf{E}+\mathbf{v} \times \mathbf{B}=\eta \mathbf{j}$, the evolution of a magnetic field in a plasma flow is described by the induction equation [1],

$$
\frac{\partial \mathbf{B}}{\partial t}=\nabla \times \mathbf{v} \times \mathbf{B}+\frac{\eta}{\mu_{0}} \nabla^{2} \mathbf{B},
$$

where $\mathbf{v}(\mathbf{x}, t)$ is the flow field of a plasma with resistivity $\eta$, and $\mu_{0}$ is the permeability of free space. The solution to this equation for a given flow field would provide an answer to the kinematic dynamo problem and further constrain the self-consistent magnetohydrodynamic (MHD) dynamo theory. The Ohmic heating of a plasma $\left(\eta j^{2}\right.$, with $\left.\mathbf{j}=\nabla \times \mathbf{B}\right)$ can also be understood from the solution to this equation.

Traditional [2] and helicity conserving [3-5] dynamo theories presume a turbulent background conducting flow. A two scale approach [6] is applied, which decomposes the flow field and the magnetic field into a mean and a fluctuating component. A quasi-linear approximation then gives rise to an analytic closed form for the time evolution of the mean field, the so-called $\alpha-\beta$ turbulent dynamo theory. If the mean field of the flow velocity is spatially nonuniform, like the differential rotation envisioned on the sun, another effect, the so-called $\Omega$ term, presents and plays the role of converting a poloidal field into a toroidal one.

Phenomenologically speaking, the build-up of a large scale magnetic field by dynamo action corresponds to stretching the magnetic flux tubes and piling them up in a noncancellating manner. This was pictorially illustrated by Alfvén's twist model [7]. The condition for exponential magnetic field growth in the dissipationless limit is made rigorous mathematically by the criteria that the flow has chaotic Lagrangian trajectories, a point that was first made precise by Arnold, et al [8]. The so-called chaotic dynamo has since then received much attention and attempted by many authors (for a list, see reference [9]).

There are a number of aspects that distinguish a chaotic dynamo from a turbulent one. First, although turbulent flows generally have chaotic Lagrangian trajectories, chaotic flows 
need not be turbulent. Smooth laminar flows with velocity field of the spatial scale of the system size are well-known to have chaotic or non-integrable trajectories. By an analog to a theorem in Hamiltonian mechanics, it is indeed an exceptional case for a divergencefree Laminar flow to be integrable. Second, the shift of emphasis to smooth laminar flow precludes the two-scale analysis like the one in the $\alpha$ - $\beta$ turbulent dynamo theory. On the other hand, the well-behaveness of the flow field allows some rigorous results in the dissipationless limit for a general flow, the so-called Cauchy solution. The connection to dynamical system theory provides a new set of tools to characterize the problem [9].

The chaotic and turbulent dynamo theories share a common challenge that complicates a fundamental understanding of the problem, which is the natural concentration of magnetic energy growth in small scale fields. In the $\alpha-\beta$ turbulent dynamo theory, this effect comes in by imposing a much shorter time for the range of validity of the theory than what originally had been hoped [10]. The reason is that the small scale fluctuating field reaches energy equipartition with the flow much earlier than the mean field [11]. In other words, it manifests itself as a violation of the (quasi-linear) approximation. In the chaotic dynamo problem, no approximation was made but one faces even more obvious difficulties. For example, the eigenmode expansion series for the magnetic field in the case of a perfectly conducting chaotic divergence-free flow, diverges at the high wave-number end of the spectrum at large time, an example of the so-called 'ultra-violet' catastrophe. A finite resistivity would in principle provide a cut-off for this 'ultra-violet' divergence. A straightforward scaling argument shows that the smallest structure, or the inverse of the largest wave-vector of the cut-off eigenmode, scales as $R_{m}^{-1 / 2}$, with $R_{m} \equiv \mu_{0} L V / \eta$ the magnetic Reynolds number ( $L$ is the characteristic spatial scale, $V$ is the typical flow speed, and $\eta$ is the resistivity). In astrophysical situations where the dynamo theory is primarily applied, the magnetic Reynolds number is of the order $10^{8}$ or higher. Just like the fully-developed fluid turbulence problem, it is still beyond the reach of a direct numerical simulation.

The causes of small scale structures in a turbulent dynamo and a chaotic one appear to be different. In a turbulent dynamo, the small scale structures for the magnetic field are 
usually said to be associated with the small scale structures of the fluctuating flow velocity field. This is certainly not a viable explanation for a chaotic dynamo in which the flow field can be laminar and possesses a spatial scale comparable to system size. Instead the fine structures and the extreme anisotropies are entirely determined by the kinematics of the flow trajectories. The purpose of this paper is to illustrate how the complicated spatial structures in the magnetic field evolution can be understood in terms of the finite time Lyapunov exponent that describes the behavior of neighboring points in a chaotic flow.

The basic features can be illustrated by following the relative motion between two nearby flow elements in an ideal evolution, i.e. the frozen flux limit. By definition, in a chaotic flow, neighboring flow elements generally separate exponentially apart in time. If the two flow elements align perfectly along the so-called stable direction, they would actually converge exponentially in time. The conjugacy of exponential convergence and divergence can be trivially explained in a divergence-free flow. It actually holds true for any flow as long as the divergence of the flow field does not diverge exponentially. If the initial fields carried by the flow elements are different, exponential convergence implies an exponentially increasing gradient of the magnetic field, which is equivalent to an exponential reduction of the spatial scale of the magnetic field along the stable direction. Similarly along the unstable direction, exponential divergence would imply that the field would be smoothed out and develop an exponentially increasing spatial scale (We will show later this actually does not happen in a typical flow due to the spatial anisotropies of the local Lyapunov exponents). The so-called coordinate contraction (dilation) effect is one of the two ways to significantly alter the spatial scales of the magnetic field and the current density distribution in a chaotic flow. The other effect is the so-called differential growth.

The flow elements, while undergo a relative motion, are simultaneously distorted by the flow motion. In a chaotic flow, initial blobs would be stretched into thin stripes. The frozen flux is therefore squeezed and gives rise to an exponential growth of the magnetic field strength. The growth rate of the field is also given by the local Lyapunov exponent. Since the local Lyapunov exponent is a function of position (where the fiducial Lagrangian 
trajectory starts) and time (how long the fiducial trajectory is traced). The spatial variation of the local Lyapunov exponent, more specifically the time dependence of the spatial gradient of the local Lyapunov exponent, plays an equally, if not more important role, in determining the field structure and current distribution. Along the unstable direction, the local Lyapunov exponent varies wildly, differential growth dominates coordinate dilation, and leads to an algebraic variation of the field spatial scales. In the stable direction, the local Lyapunov exponent varies smoothly and the scale reduction rate remains to be the local Lyapunov exponent. However, even if the initial field is uniform, the differential growth, with the help of coordinate contraction, still induces an exponential scale reduction.

The observation of increasingly fine structures in chaotic, high magnetic Reynolds number flows, is widely reported in numerical simulations. It is generally thought that within the kinematic dynamo approximation, the kinematic magnetic fields would concentrate on a fractal set in space in the limit of $R_{m} \rightarrow \infty$ and $t \rightarrow \infty$. The most notable analysis in support of this is due to Ott, et al [12]. More specifically, these authors showed that a magnetic field, stretched according to the two dimensional baker's map and folded back in a reinforcing manner with the help of field line cutting, would concentrate on a fractal set in a two dimensional space in the limit of $t \rightarrow \infty$. Although this analysis is illuminating, particularly for understanding the long time limit of the idealized, so-called Stretch-twist-fold kinematic dynamo model, it does suffer from two unphysical assumptions. First, the two dimensional baker's map, like other hyperbolic systems, can not be reduced from a physically realisable flow. Second, field line cutting is an unphysical operation due to the absence of magnetic monopole. Since unphysical assumptions could lead to unphysical results, more rigorous analysis without these unphysical assumptions is clearly desirable.

Our analysis removes the constraints of the two unphysical assumptions, and also has a different emphasis. We will try to understand the time evolution of how structures and anisotropies develop in a conducting flow, and what determines the time evolution, as supposed to the time asymptotic limits of the kinematic approximation. The knowledge of the field and current distribution also allows us to study the effect of resistive diffusion and the 
back reaction of the Lorenz force, i.e. the breakdown of the kinematic approximation. Both the characteristic directions of the Lagrangian flow trajectory and the finite time Lyapunov exponent are shown to be the central concepts that relate the flow to the magnetic field evolution. Unlike many authors in the past [13], we go beyond merely using finite time Lyapunov exponents as a statistical quantity to characterize transport. We will, for the first time, use the spatial and temporal dependence of the finite time Lyapunov exponent to rigorously examine the spatial and temporal evolution of the magnetic field and current distribution. We discovered [14,15] that the finite time Lyapunov exponent can be described by two convergence functions with drastically different properties. The strongly anisotropic behavior of the convergence functions for the finite time Lyapunov exponent, is found to be crucial to understand the spatial structures of the field and current distribution. This is clearly demonstrated in the discussions which weigh the relative importance of coordinate contraction and differential growth for scale reduction in section III.

The main body of the paper is organized as follows. The mathematical background for the analysis is given in section II. In particular, section II A gives an interpretation of Oseledec's multiplicative ergodic theory [20] in terms of a mapping between Lagrangian and Eulerian coordinates. The characteristic directions that describe the spatial anisotropies in both Lagrangian and Eulerian frames, are introduced in section II B, along with some basic calculus of coordinate transformations. Section II C summarizes some of the generic features of the local or finite time Lyapunov exponents, with an emphasis on its spatial variation. Two special cases of the magnetic field evolution problem, the ideal evolution in three dimensions and the non-ideal evolution in two dimensions, are then presented. One is the ideal evolution in three dimensions, the other is the non-ideal evolution of a two dimensional field. The ideal case, section III, is intended to illustrate the small scale field structures. The non-ideal case, section IV, is to demonstrate the time scale and the magnitude of Ohmic dissipation. Ohmic heating in a chaotic plasma naturally concentrates in either filaments, ribbon, or sheets, and the condition that determines which of these forms occurs is given in section $\mathrm{V}$. The competition between field growth, Ohmic dissipation, and the back-reaction 
of the Lorentz force, is discussed in section VI. The preferred direction of the Lorentz force is also pointed out there.

\section{THE ANISOTROPIES OF LAGRANGIAN CHAOS}

\section{A. Lagrangian chaos in Lagrangian coordinates}

The trajectory $\mathrm{x}(\xi, t)$ of a flow point starting from position $\xi$ at initial time is followed by integrating

$$
\left(\frac{\partial \mathbf{x}}{\partial t}\right)_{\xi}=\mathbf{v}(\mathbf{x}, t)
$$

With the typical choice of $\mathrm{x}(\xi, t=0)=\xi, \xi$ is known as the Lagrangian coordinates in fluid mechanics. The practical definition of chaos, i.e., the sensitive dependence of the final state $[\mathrm{x}(\xi, t)]$ on its initial condition $[\xi]$, can be interpreted in terms of a mapping between the Lagrangian coordinates $\xi$ and the ordinary position vector $\mathbf{x}$ in Eulerian frame through the functional relationship $\mathrm{x}(\xi, t)$, which is obtained by integrating equation (2).

The chaotic behavior is quantitatively described by the exponential rate at which an initial error or displacement is amplified over time. This information is given by the metric tensor of the Lagrangian coordinates, a point that can be seen by expressing the differential distance in the Eulerian frame $(d l)^{2}=d \mathbf{x} \cdot d \mathbf{x}$ in Lagrangian coordinates using the chain rule,

$$
(d l)^{2}=d \mathbf{x} \cdot d \mathbf{x}=\sum_{i} \frac{\partial \mathbf{x}}{\partial \xi^{i}} d \xi^{i} \cdot \sum_{j} \frac{\partial \mathbf{x}}{\partial \xi^{j}} d \xi^{j}=\sum_{i j} g_{i j} d \xi^{i} d \xi^{j},
$$

with $g_{i j} \equiv\left(\partial \mathbf{x} / \partial \xi^{i}\right) \cdot\left(\partial \mathbf{x} / \partial \xi^{j}\right)$ the metric tensor of the Lagrangian coordinates. The matrix inverse of $g_{i j}$, which is $g^{i j} \equiv \nabla \xi^{i} \cdot \nabla \xi^{j}$, is also called the metric tensor of the Lagrangian coordinates. The two representations of the metric tensor are associated with the freedom of expressing an arbitrary vector $\mathbf{A}$ in either covariant representation $\mathbf{A}=\sum_{i} A_{i} \nabla \xi^{i}$ or contravariant representation $\mathbf{A}=\sum A^{i}\left(\partial \mathbf{x} / \partial \xi^{i}\right)$.

The description of the kinematics of chaos in Lagrangian coordinates becomes obvious once the metric tensor of the Lagrangian coordinates $g_{i j}$ is interpreted using the language of 
dynamical systems as the Oseledec matrix $\Lambda_{i j}$ of Oseledec's multiplicative ergodic theorem $[20,19]$.

Since $g_{i j}$ is a positive definite and symmetric matrix, it can be diagonalized with positive eigenvalues and real eigenvectors,

$$
g_{i j}=\Lambda_{l} e_{i} e_{j}+\Lambda_{m} m_{i} m_{j}+\Lambda_{s} s_{i} s_{j},
$$

with the three eigenvalues $\Lambda_{l} \geq \Lambda_{m} \geq \Lambda_{s}>0$. The metric tensor determines the distance between neighboring flow points, so this tensor determines the three (finite time or local) Lyapunov exponents,

$$
\lambda_{l}(\xi, t) \equiv \frac{\ln \Lambda_{l}}{2 t}, \lambda_{m}(\xi, t) \equiv \frac{\ln \Lambda_{m}}{2 t}, \lambda_{s}(\xi, t) \equiv \frac{\ln \Lambda_{s}}{2 t}
$$

which are functions of both position and time. A flow is chaotic if the largest Lyapunov exponent $\lambda_{l}$ has a non-zero limit as time goes to infinity. Otherwise it is said to be integrable.

Chaotic motion of a flow point is said to be of Lagrangian nature in that 1] it is most easily interpreted as a mapping between the Lagrangian coordinates and the ordinary position vector in Eulerian frame, and 2] the kinematics of chaos, most notably the local Lyapunov

exponents [see equation (4)] and the characteristic (un)stable directions [see section (II B)], are naturally defined as functions of the Lagrangian coordinates (initial conditions). The physical observable, like the magnetic field, induced current density, and Ohmic heating, however, are usually followed in an Eulerian frame. The theory of coordinate transformations provides the link that relates the Eulerian physical observable to the kinematics of chaos in Lagrangian coordinates, which is the main focus of this paper.

\section{B. Lagrangian and Eulerian characteristic directions for the anisotropic transport}

As we will show, the magnetic field in a chaotic flow evolves remarkably differently in the various characteristic directions of the flow. Here we introduce the characteristic directions that will be required to calculate quantities such as the magnetic flux concentration, the 
current channel, and the Ohmic heating in the magnetic field evolution problem. The basic theory of general coordinates, upon which the later sections will be heavily drawn, are also outlined here. As the title of the section suggests, two sets of characteristic directions, one in Lagrangian frame and the other in the Eulerian frame, will be introduced. The construction of characteristic directions in Eulerian frame is based on their Lagrangian counterparts, so we will start with the eigenvectors of the metric tensor of the Lagrangian coordinates.

The real eigenvectors of the matrix $g_{i j}$, equation (3), defines two sets of vectors: three orthonormal vectors $\hat{\mathbf{e}} \equiv\left\{e_{i}\right\}, \hat{\mathbf{m}} \equiv\left\{m_{i}\right\}, \hat{\mathbf{s}} \equiv\left\{s_{i}\right\}$ in Lagrangian frame and three orthogonal vectors $\mathbf{e} \equiv e_{i} \nabla \xi^{i}, \mathbf{m} \equiv m_{i} \nabla \xi^{i}, \mathbf{s} \equiv s_{i} \nabla \xi^{i}$ in Eulerian frame. The orthonormal vectors $\hat{\mathbf{e}}, \hat{\mathbf{m}}, \hat{\mathbf{s}}$ in Lagrangian frame have well-defined time-asymptotic limits that are generally position dependent, $\hat{\mathbf{e}}_{\infty}(\xi)=\left\{e_{i}^{\infty}(\xi)\right\}, \hat{\mathbf{m}}_{\infty}(\xi)=\left\{m_{i}^{\infty}(\xi)\right\}, \hat{\mathbf{s}}_{\infty}(\xi)=\left\{s_{i}^{\infty}(\xi)\right\}$. In the case of $\lambda_{l}^{\infty}=$ $-\lambda_{s}^{\infty}>0$ and $\lambda_{m}^{\infty}=0$, neighboring points diverge (converge) exponentially in time if initially separated along $\hat{\mathbf{e}}_{\infty}\left(\hat{\mathbf{s}}_{\infty}\right)$ direction, but their distance varies at most algebraically in time if initially separated along the $\hat{\mathbf{m}}_{\infty}$ direction. The convergence of $\hat{\mathbf{e}}, \hat{\mathbf{m}}, \hat{\mathbf{s}}$ to their time asymptotic limits $\hat{\mathbf{e}}_{\infty}, \hat{\mathbf{m}}_{\infty}, \hat{\mathbf{s}}_{\infty}$ is usually exponential in chaotic regions. In the case of a two dimensional divergence-free chaotic flow, one can write [14]

$$
\hat{\mathbf{e}} \propto \hat{\mathbf{e}}_{\infty}+(\sigma / \Lambda) \hat{\mathbf{s}}_{\infty} ; \hat{\mathbf{s}} \propto \hat{\mathbf{s}}_{\infty}-(\sigma / \Lambda) \hat{\mathbf{e}}_{\infty}
$$

with $\Lambda \geq 1$ the eigenvalue of the metric tensor and $\sigma$ an algebraic function measuring the convergence rate.

The contravariant representation of the metric tensor, $g^{i j} \equiv \nabla \xi^{i} \cdot \nabla \xi^{j}$, is the matrix inverse of $g_{i j}$ and has the diagonal form, $g^{i j}=\Lambda_{l}^{-1} E^{i} E^{j}+\Lambda_{m}^{-1} M^{i} M^{j}+\Lambda_{s}^{-1} S^{i} S^{j}$. The eigenvectors of the matrix $g^{i j}$ are exactly the same as those of $g_{i j}$, so the orthonormal vectors $\hat{\mathbf{E}} \equiv\left\{E^{i}\right\}, \hat{\mathbf{M}} \equiv\left\{M^{i}\right\}, \hat{\mathbf{S}} \equiv\left\{S^{i}\right\}$ in Lagrangian frame are identical to $\hat{\mathbf{e}}, \hat{\mathbf{m}}, \hat{\mathbf{s}}$. The orthogonal vectors $\mathbf{E} \equiv E^{i} \partial \mathbf{x} / \partial \xi^{i}, \mathbf{M} \equiv M^{i} \partial \mathbf{x} / \partial \xi^{i}, \mathbf{S} \equiv S^{i} \partial \mathbf{x} / \partial \xi^{i}$ in Eulerian frame are parallel to e, m, s.

Using the time asymptotic limits $s_{i}^{\infty}(\xi)$ and $E_{\infty}^{i}(\xi)$ one can construct three new orthogonal vectors in Eulerian frame, 


$$
\mathcal{S} \equiv s_{i}^{\infty} \nabla \xi^{i}, \quad \mathcal{E} \equiv E_{\infty}^{i} \partial \mathrm{x} / \partial \xi^{i}, \quad \mathcal{M} \equiv \mathcal{S} \times \mathcal{E} .
$$

The vectors $\mathbf{e}(\mathbf{E}), \mathbf{m}(\mathbf{M}), \mathbf{s}(\mathbf{S})$ converge exponentially to $\mathcal{E}, \mathcal{M}$, and $\mathcal{S}$ in directions. The three vector directions as given by $\left\{\hat{\mathbf{e}}_{\infty}, \hat{\mathbf{m}}_{\infty}, \hat{\mathbf{s}}_{\infty}\right\}$ in Lagrangian frame and $\{\mathcal{E}, \mathcal{M}, \mathcal{S}\}$ in Eulerian frame are useful to understand the extreme spatial anisotropy of the chaotic transport of a scalar or vector field. Along with the finite time Lyapunov exponents, they determine much of the physics of magnetic field evolution.

Next we give two explicit demonstrations that are helpful to those who wish to reproduce the details of the calculations in the later sections. In particular, we show how quantities in Eulerian frame are related to those expressions in Lagrangian coordinates. For example the inner product of $\mathbf{E}$ with itself is

$$
\begin{aligned}
\mathbf{E} \cdot \mathbf{E} & =E^{i} \partial \mathbf{x} / \partial \xi^{i} \cdot E^{j} \partial \mathbf{x} / \partial \xi^{j} \\
& =E^{i} g_{i j} E^{j} \\
& =E^{i}\left(\Lambda_{l} e_{i} e_{j}+\Lambda_{m} m_{i} m_{j}+\Lambda_{s} s_{i} s_{j}\right) E^{j} \\
& =\Lambda_{l},
\end{aligned}
$$

which grows exponentially in time for a chaotic flow. A common expression that will be evaluated in later sections is of the form $\mathcal{E} \cdot \nabla f$ with $f$ some function of position. By the chain rule, one has

$$
\nabla f=\left(\partial f / \partial \xi^{i}\right) \nabla \xi^{i}
$$

The orthogonal relation

$$
\frac{\partial \mathbf{x}}{\partial \xi^{i}} \cdot \nabla \xi^{j}=\delta_{i}^{j}
$$

is another trivial result of the chain rule. Combining these two, one finds

$$
\begin{aligned}
\mathcal{E} \cdot \nabla f & =E_{\infty}^{i}\left(\partial \mathbf{x} / \partial \xi^{i}\right) \cdot\left(\partial f / \partial \xi^{j}\right) \nabla \xi^{j} \\
& =E_{\infty}^{i} \delta_{i}^{j}\left(\partial f / \partial \xi^{j}\right) \\
& =E_{\infty}^{i}\left(\partial f / \partial \xi^{i}\right) \\
& =\hat{\mathbf{e}}_{\infty} \cdot \nabla_{0} f
\end{aligned}
$$


where $\nabla_{0}$ denotes a gradient in Lagrangian coordinates.

\section{Properties of the local Lyapunov exponents}

The local Lyapunov exponents are defined in equation (4) through the metric tensor of the Lagrangian coordinates, which are properties of the flow field alone. In other words, once the flow field is specified, the local Lyapunov exponents are completely determined, as functions of the initial position (i.e. the Lagrangian coordinates) and time. Although an explicit calculation is required for a quantitative understanding, there are generic features for the local Lyapunov exponents that are independent of the particular form of the flow field.

The simplest case, where the local Lyapunov exponents are spatially independent constants, occurs only for purely hyperbolic systems. An example is the Arnold's cat map. All physically relevant chaotic systems found to date have non-hyperbolic points in which case the local Lyapunov exponent varies significantly over space and time. In the time asymptotic limit, the local Lyapunov exponent converges to the infinite time Lyapunov exponent, which is a constant for a given ergodic region. The spatial and time dependence of the local Lyapunov exponent are contained in the so-called convergence functions of the expressions for the local Lyapunov exponents.

With exponential accuracy, the finite time Lyapunov exponent is characterized by two convergence functions $\tilde{\lambda}(\xi)$ and $f(\xi, t)[14,15]$

$$
\lambda(\xi, t)=\tilde{\lambda}(\xi) / t+f(\xi, t) / \sqrt{t}+\lambda^{\infty},
$$

where $\lambda^{\infty}$ is the infinite time Lyapunov exponent, i.e.

$$
\lim _{t \rightarrow \infty} \lambda(\xi, t)=\lambda^{\infty}
$$

The first term in equation (9) vanishes as $t \rightarrow \infty$ since $\tilde{\lambda}(\xi)$ is a function of position alone. Equation (10) then implies that $f(\xi, t)$ is bounded by $\sqrt{t}$ so

$$
\lim _{t \rightarrow \infty} f(\xi, t) / \sqrt{t}=0
$$


An analytical argument for this decomposition of the convergence functions was given in reference [14] for a two dimensional divergence-free flow. Numerical evidence was provided for three dimensional systems in reference [15].

The spatial anisotropy of the convergence functions plays a large role in determining the anisotropies of the magnetic field evolution in a chaotic plasma flow. Since the local Lyapunov exponent and the convergence functions are functions of Lagrangian coordinates, the anisotropies will be explained using the characteristic directions in Lagrangian frame. As we will show, the spatial anisotropies in Eulerian frame are straightforwardly related to the spatial anisotropies of the local Lyapunov exponents in Lagrangian frame.

The first characteristic direction we will consider is the $\hat{\mathbf{s}}_{\infty}$, the so-called stable direction along which neighboring point converge exponentially in time. Both convergence functions are well-behaved along this direction, so is the variation of the local Lyapunov exponent. In particular, $f(\xi, t)$ does not vary along this direction,

$$
\hat{\mathbf{s}}_{\infty} \cdot \nabla_{0} f(\xi, t)=0 .
$$

The variation of $\tilde{\lambda}(\xi)$ along the $\hat{\mathbf{s}}_{\infty}$ direction is smooth and related to the divergence of $\hat{\mathbf{s}}_{\infty}$, $[14,15]$

$$
\hat{\mathbf{s}}_{\infty} \cdot \nabla_{0} \tilde{\lambda}(\xi)+\nabla_{0} \cdot \hat{\mathbf{s}}_{\infty}=0
$$

Another way to look at equation (11) is that the vector field $e^{\bar{\lambda}(\xi)} \hat{\mathbf{s}}_{\infty}(\xi)$ is divergence-free, which itself may be described by a Hamiltonian [21]. The smoothness of $\hat{\mathbf{s}}_{\infty}$ implies that $\tilde{\lambda}(\xi)$ is a smooth function of position.

It is actually more widely known that the local Lyapunov exponent varies wildly over space and time, which makes the smooth variation along the $\hat{\mathbf{s}}_{\infty}$ a counter-intuitive result. The requirement for $\lambda(\xi, t)$ to vary wildly comes from the fact that the higher spatial derivatives of the Jacobian (or the metric tensor of the Lagrangian coordinates), or equivalently the local Lyapunov exponent, can be used to define the most positive infinite time Lyapunov exponents of a smooth dynamical system (see, for example, reference [16]). That is to say: 


$$
\lim _{t \rightarrow \infty} \frac{\ln \left(\nabla_{0} \lambda\right)^{2}}{2 t}=\lambda^{\infty}
$$

The $\hat{\mathbf{s}}_{\infty}$ component of $\nabla_{0} \lambda$ certainly does not satisfy equation (12). The $\hat{\mathbf{e}}_{\infty}$ component of $\nabla_{0} \lambda$ has to satisfy equation (12) since it is the least constrained direction. The $\hat{\mathbf{m}}_{\infty}$ component of $\nabla_{0} \lambda$ may or may not satisfy equation (12), as suggested by numerical calculations. This uncertainty plays a large role in determining the natural state of the concentration for the magnetic field and current heating, which includes filaments, ribbon, and sheets. Since $\tilde{\lambda}(\xi)$ is a smooth function of position and $\nabla_{0} \tilde{\lambda}(\xi)$ does not depend on time, it is $f(\xi, t)$ that describes the wild spatial variation of the local Lyapunov exponent.

\section{IDEAL EVOLUTION OF A MAGNETIC FIELD}

Within the framework of MHD, the evolution of a magnetic field embedded in a moving plasma is described by the induction equation, equation (1). For a high temperature plasma the resistivity $\eta$ is small. Hence for times less than a diffusion time (to be specified later) the temporal behavior of the magnetic field resembles an ideal evolution

$$
\partial \mathbf{B} / \partial t=\nabla \times(\mathbf{v} \times \mathbf{B}) .
$$

It has been shown $[17,18]$ that in Lagrangian coordinates, the vector potential of this field is $\mathbf{A}=\sum A_{i}(\xi) \nabla \xi^{i}$. The covariant components of the vector potential, $A_{i}(\xi)$, are independent of time in Lagrangian coordinates. By taking the curl of the vector potential to obtain the magnetic field $\mathbf{B}$ and the curl of the magnetic field to obtain the current density $\mathbf{j}$, one finds that the scalar quantities $B^{2}, j^{2}$, and $\mathbf{j} \cdot \mathbf{B}$ can be evaluated given the initial magnetic field $\mathbf{B}(\mathrm{x}, 0)$ and the metric tensor of the Lagrangian coordinates [18]. For example,

$$
\begin{aligned}
B^{2} & =\sum b^{i}(\xi) g_{i j} b^{j}(\xi) / J^{2}, \\
\mu_{0}^{2} j^{2} & =\sum\left[\frac{\partial}{\partial \xi^{n}} \epsilon^{i n m} \frac{g_{m l}}{J} b^{l}\right]\left[\frac{\partial}{\partial \xi^{p}} \epsilon^{j p q} \frac{g_{q k}}{J} b^{k}\right] \frac{g_{i j}}{J^{2}}, \\
\mu_{0} \mathbf{j} \cdot \mathbf{B} & =\sum\left[\frac{\partial}{\partial \xi^{n}} \epsilon^{i n m} \frac{g_{m l}}{J} b^{l}\right] \frac{g_{i j}}{J^{2}} b^{j} .
\end{aligned}
$$


Here $J$ is the Jacobian of the Lagrangian coordinates $\left(J^{2}=\left\|g_{i j}\right\|\right), \mu_{0}$ is the permeability of free space, and $b^{i}(\xi)=\sum \epsilon^{i j k}\left(\partial A_{k} / \partial \xi^{j}\right)$. The quantities $b^{i}(\xi)$ are just the three Cartesian components of the initial magnetic field using the initial condition for Lagrangian coordinates $\mathrm{x}(\xi, 0)=\xi$.

In a region where the flow is chaotic $\left(\lambda_{l}^{\infty}>0\right)$, both the magnetic energy and the induced current density increase exponentially in time

$$
\begin{aligned}
B^{2} & =\left(\mathbf{B}_{0} \cdot \hat{\mathbf{e}}_{\infty}\right)^{2} \Lambda_{l}+O\left(\Lambda_{m}\right) \\
& \approx\left(\mathbf{B}_{0} \cdot \hat{\mathbf{e}}_{\infty}\right)^{2} \exp \left(2 \lambda_{l} t\right) \\
\mu_{0}^{2} j^{2} & =\Lambda_{l}^{3}\left(\mathbf{B}_{0} \cdot \hat{\mathbf{e}}_{\infty}\right)^{2}\left[\hat{\mathbf{e}}_{\infty} \cdot\left(\nabla_{0} \times \hat{\mathbf{e}}_{\infty}\right)\right]^{2}+O\left(e^{4 \lambda_{l} t}\right) \\
& \propto \exp \left(6 \lambda_{l} t\right)
\end{aligned}
$$

The leading term in the current density has no dependence on the spatial derivative of the initial magnetic field with respect to Lagrangian coordinates, but the next order terms do include terms of that kind, see appendix A for detail. The induced current density tends to align with the magnetic field in a chaotic flow since

$$
\mu_{0} \mathbf{j} \cdot \mathbf{B}=\Lambda_{l}^{2}\left(\mathbf{B}_{0} \cdot \hat{\mathbf{e}}_{\infty}\right)^{2}\left[\hat{\mathbf{e}}_{\infty} \cdot\left(\nabla_{0} \times \hat{\mathbf{e}}_{\infty}\right)\right]+O\left(\Lambda_{l}\right)
$$

which implies $(\mathbf{j} \cdot \mathbf{B})^{2} / j^{2} B^{2} \approx 1+\exp \left(-2 \lambda_{l} t\right)$. The alignment of the magnetic field and the current density is fundamentally dependent on the flow being truly three dimensional, which means that $\hat{\mathbf{e}}_{\infty} \cdot \nabla_{0} \times \hat{\mathbf{e}}_{\infty}$ does not identically vanish. For a two dimensional flow of the form $\mathbf{v}=\hat{\mathbf{z}} \times \nabla \psi(x, y, t), \hat{\mathbf{e}}_{\infty}$ is confined to the $x-y$ plane and $\nabla \times \hat{\mathbf{e}}_{\infty}$ lies along the $\hat{\mathbf{z}}$ axis. Hence $\hat{\mathbf{e}}_{\infty} \cdot \nabla_{0} \times \hat{\mathbf{e}}_{\infty}$ identically vanishes and the current flows along the $\hat{\mathbf{z}} \operatorname{direction}(\mathbf{j} \perp \mathbf{B})$ with the leading terms in $j^{2}$ of time dependence $\exp \left(4 \lambda_{l} t\right)$, see appendix $\mathrm{A}$.

The exact spatial decomposition of the magnetic field and the current density requires the knowledge of vectors $\mathcal{E}, \mathcal{M}, \mathcal{S}$, the Eulerian frame correspondences of vectors $\hat{\mathbf{e}}_{\infty}, \hat{\mathbf{m}}_{\infty}, \hat{\mathbf{s}}_{\infty}$, see section II B for precise definitions. The contravariant representation of an ideally evolving magnetic field is

$$
\mathbf{B}=\frac{1}{J} \sum_{i} b^{i}(\xi) \frac{\partial \mathbf{x}}{\partial \xi^{i}}
$$


with $b^{i} \equiv \sum_{j k} \epsilon^{i j k} \partial A_{k} / \partial \xi^{j}$. This expression is equivalent to the Cauchy solution to the ideal induction equation. The spatial decomposition of $\mathbf{B}$ can be found by dotting $\mathbf{B}$ with the characteristic directions in the Eulerian frame.

The magnetic field lies essentially along the $\mathcal{E}$ direction in the Eulerian frame or the real space,

$$
\begin{aligned}
B_{e} & \equiv \frac{\mathcal{E} \cdot \mathbf{B}}{|\mathcal{E}|} \approx\left(\mathbf{B}_{0} \cdot \hat{\mathbf{e}}_{\infty}\right) \sqrt{\Lambda_{l}} \\
B_{m} & \equiv \frac{\mathcal{M} \cdot \mathbf{B}}{|\mathcal{M}|} \approx\left(\mathbf{B}_{0} \cdot \hat{\mathbf{m}}_{\infty}\right) \sqrt{\Lambda_{m}} \\
B_{s} & \equiv \frac{\mathcal{S} \cdot \mathbf{B}}{|\mathcal{S}|} \approx\left(\mathbf{B}_{0} \cdot \hat{\mathbf{s}}_{\infty}\right) \sqrt{\Lambda_{s}}
\end{aligned}
$$

since $\sqrt{\Lambda_{l}}=e^{\lambda_{l} t} \gg \sqrt{\Lambda_{m}}=e^{\lambda_{m} t} \gg \sqrt{\Lambda_{s}}=e^{\lambda_{s} t}$ for $\lambda_{l}>\lambda_{m}>\lambda_{s}$.

The current density has a similar spatial anisotropy. It suffices to note here that $j_{e} \propto$ $e^{3 \lambda t} \gg j_{m} \propto e^{2 \lambda t}$. The exact time dependence of the $\mathcal{S}$ component $j_{s}$ can be either $\propto e^{\lambda t}$ or $\propto e^{2 \lambda t}$, depending on whether $\hat{\mathbf{m}}_{\infty} \cdot \nabla_{0} \lambda(\xi, t)$ exponentiates in time or not. The detailed form of the current distribution will be considered in section $\mathrm{V}$.

As time progresses, the magnetic field develops structures of different spatial scales along the $\mathcal{E}, \mathcal{M}$, and $\mathcal{S}$ directions. The gradient of the magnetic pressure along $\mathcal{S}$ direction is

$$
\begin{aligned}
& \frac{\mathcal{S} \cdot \nabla B^{2}}{|\mathcal{S}|}=\frac{\hat{\mathbf{s}}_{\infty} \cdot \nabla_{0} B^{2}}{\sqrt{\Lambda_{s}}}+O\left(\Lambda_{s}^{-1 / 2}\right) \\
& =\frac{B^{2}}{\sqrt{\Lambda_{s}}} 2 \hat{\mathbf{s}}_{\infty} \cdot \nabla_{0}\left(\lambda_{l} t\right)+\frac{B^{2}}{\sqrt{\Lambda_{s}}} \cdot \frac{\hat{\mathbf{s}}_{\infty} \cdot \nabla_{0}\left[\left(\mathbf{B}_{0} \cdot \hat{\mathbf{e}}_{\infty}\right)^{2}\right]}{\left(\mathbf{B}_{0} \cdot \hat{\mathbf{e}}_{\infty}\right)^{2}} \\
& +O\left(\Lambda_{s}^{-1 / 2}\right) \text {. }
\end{aligned}
$$

As before, $\nabla_{0}$ denotes gradient in the Lagrangian frame. Hence the magnetic pressure develops an exponentially decreasing spatial scale $\left(\lambda_{s}<0\right)$ along the $\mathcal{S}$ lines,

$$
l_{s} \equiv\left[\frac{\mathcal{S} \cdot \nabla B^{2}}{|\mathcal{S}| B^{2}}\right]^{-1} \approx l_{s}^{0} \exp \left(\lambda_{s} t\right)
$$

The exact contribution for $l_{s}^{0}$

$$
l_{s}^{0}=\left\{2 \hat{\mathbf{s}}_{\infty} \cdot \nabla_{0}\left(\lambda_{l} t\right)+\frac{\hat{\mathbf{s}}_{\infty} \cdot \nabla_{0}\left[\left(\mathbf{B}_{0} \cdot \hat{\mathbf{e}}_{\infty}\right)^{2}\right]}{\left(\mathbf{B}_{0} \cdot \hat{\mathbf{e}}_{\infty}\right)^{2}}\right\}^{-1}
$$


requires some clarification. The expressions in Lagrangian coordinates (frame) clarify two separate mechanisms for scale reduction of the magnetic field. The first is coordinate contraction, which is represented by the second term in equation (23). The other one comes from differential growth, which corresponds to the first term in equation (23). The two mechanisms are of comparable importance, or equivalently speaking, the two terms in equation (23) are of the same order only because the finite time Lyapunov exponent varies smoothly along the $\hat{\mathbf{s}}_{\infty}$ direction so $\hat{\mathbf{s}}_{\infty} \cdot \nabla_{0} \lambda_{l} t$ does not have a time dependence. Physically the second term (coordinate contraction) describes the contribution from an initial field distribution with spatial variation. The first term (differential growth) is the contribution from the flow alone. The obvious explanation is that a general chaotic flow with $\lambda_{l}$ spatially non-uniform can generate spatially non-uniform magnetic field even for a uniform initial seed field. A less obvious point is that $\lambda_{l}$ does not vary wildly in the $\hat{\mathbf{s}}_{\infty}$ direction so this contribution does not overshadow the second term. For example, had $\hat{\mathbf{s}}_{\infty} \cdot \nabla_{0}\left(\lambda_{l} t\right)$ behaved as $\hat{\mathbf{e}}_{\infty} \cdot \nabla_{0}\left(\lambda_{l} t\right)$ does, $l_{s}$ would have a time dependence of $\exp \left(\lambda_{s}-\lambda_{l}\right) t$. This is a point that has not been clarified in the literature.

Three limiting cases can occur. The first is that both $\lambda_{l}$ and $\mathbf{B}_{0} \cdot \hat{\mathbf{e}}_{\infty}$ are independent of position, then the magnetic field can never develop exponentially decreasing spatial scales. The second case is that only $\lambda_{l}$ is independent of position, then the field structures shrink exponentially in time $\left(e^{\lambda_{s} t}\right)$ due to the converging Lagrangian trajectories bringing neighboring magnetic field of different magnitude exponentially close along the $\hat{\mathbf{s}}_{\infty}$ direction, the so-called coordinate contraction factor. The third case is that the initial magnetic field is spatially uniform so coordinate contraction makes no contribution, the small scale structures for the magnetic field come from the (smooth) spatial variation of $\lambda_{l}$ along the $\hat{\mathbf{s}}_{\infty}$ direction, the so-called differential growth factor.

The field line lies basically along the $\mathcal{E}$ direction and naturally has the least spatial structure in this direction. The usual pictorial analysis says that Lagrangian trajectories carrying different $\mathbf{B}$ s are exponentially separating apart in the $\hat{\mathbf{e}}_{\infty}$ direction so the characteristic scale of the field should increase exponentially in time. An exact calculation shows this is not 
the case in general after taking into account the spatial variation of the local Lyapunov exponent. To leading order,

$$
\frac{\mathcal{E} \cdot \nabla B^{2}}{|\mathcal{E}| B^{2}}=\frac{\hat{\mathbf{e}}_{\infty} \cdot \nabla_{0}\left(\mathbf{B}_{0} \cdot \hat{\mathbf{e}}_{\infty}\right)}{e^{\lambda_{l} t}\left(\mathbf{B}_{0} \cdot \hat{\mathbf{e}}_{\infty}\right)}+\frac{\hat{\mathbf{e}}_{\infty} \cdot \nabla_{0}\left(2 \lambda_{l} t\right)}{e^{\lambda_{l} t}} .
$$

The first term is the familiar contribution from the exponentially divergence of neighboring trajectories, which can be analogously called coordinate dilation. It becomes exponentially small in time. Except for the special case where $\lambda_{l}$ is independent of position, the second term, the differential growth factor, overtakes the first term in determining the spatial structures of the field along the $\mathcal{E}$ direction. The reason is that $\hat{\mathbf{e}}_{\infty} \cdot \nabla_{0}\left(\lambda_{l} t\right)$ has an exponential time dependence. That is, to leading order, the characteristic scale in the $\mathcal{E}$ direction is given by

$$
l_{e} \equiv\left[\frac{\mathcal{E} \cdot \nabla B^{2}}{|\mathcal{E}| B^{2}}\right]^{-1} \approx \frac{\sqrt{\Lambda_{l}}}{2 \hat{\mathbf{e}}_{\infty} \cdot \nabla\left(\lambda_{l} t\right)}
$$

The time evolution of the spatial scale of the magnetic field along the $\mathcal{E}$ direction has an algebraic dependence in time due to the fact that

$$
\lim _{t \rightarrow \infty} \ln \left[\hat{\mathbf{e}}_{\infty} \cdot \nabla(\lambda t)\right]^{2} / 2 t=\lambda^{\infty},
$$

which is generally true for flow fields with smooth spatial gradients [16]. In general one has

$$
\frac{\sqrt{\Lambda_{l}}}{\hat{\mathbf{e}}_{\infty} \cdot \nabla\left(\lambda_{l} t\right)} \propto t^{q} .
$$

If $q<0$, the spatial structure of the magnetic field along the $\mathcal{E}$ direction becomes ever finer. If $q>0$, the spatial structure of the magnetic field along the $\mathcal{E}$ lines tends to diminish under chaotic advection. A flow field with $q \geq 0$ could be favorable for sustaining a magnetic dynamo.

The leading order terms that describes the time evolution of the spatial scales of the magnetic field along the $\mathcal{M}$ direction has a similar breakdown as before. There is one term associated with the initial gradient of the magnetic field, and there is another term account for the spatial variation of the local Lyapunov exponent. Written out explicitly,

$$
\frac{\mathcal{M} \cdot \nabla B^{2}}{|\mathcal{M}| B^{2}}=\frac{\hat{\mathbf{m}}_{\infty} \cdot \nabla_{0}\left(\mathbf{B}_{0} \cdot \hat{\mathbf{e}}_{\infty}\right)}{\sqrt{\Lambda_{m}}\left(\mathbf{B}_{0} \cdot \hat{\mathbf{e}}_{\infty}\right)}+\frac{\hat{\mathbf{m}}_{\infty} \cdot \nabla_{0}\left(2 \lambda_{l} t\right)}{\sqrt{\Lambda_{m}}}
$$


In the most general case, $\lambda_{m} \equiv \ln \Lambda_{m} / 2 t$ can be any real number since even for a divergencefree flow the most stringent constraint is for $\lambda_{l}+\lambda_{m}+\lambda_{s}=0$. Usually $\lambda_{l}>0$ for a chaotic flow and $\lambda_{s}<0$ so as to be practically relevant. For a time-independent flow, $\lambda_{m}^{\infty}$ always vanishes, but the same is not always true if the flow field has a time dependence.

The spatial gradient of the initial magnetic field plays less a role in determining the time evolution of the field structure if $\lambda_{m}$ is positive. This case is similar to that of the $\mathcal{E}$ direction, in that the second term always dominates the first term in determining the time asymptotic behavior of the field structure. Equivalently speaking, differential growth rather than coordinate dilation plays the more important role if $\lambda_{m}>0$. In making this statement, we have assumed that the variation of the local Lyapunov exponent along the second unstable direction ( $\hat{\mathbf{m}}_{\infty}$ associated with a $\left.\lambda_{m}>0\right)$ is not smooth and $\hat{\mathbf{m}}_{\infty} \cdot \nabla_{0}\left(2 \lambda_{l} t\right)$ has an exponential time dependence. Although a proof is not available, we find it to be the most plausible scenario, since otherwise one arrives at the peculiar result that the field structures would flatten out at an exponential rate, a case that is not even true for the most unstable direction $\mathcal{E}$.

If $\lambda_{m}^{\infty}$ is less than zero, the flow has two contracting directions and one expanding direction. The field scale along the second stable direction $\left(\hat{\mathbf{m}}_{\infty}\right.$ with $\left.\lambda_{m}^{\infty}<0\right)$ has qualitatively the same behavior as that of $l_{s}$ along $\hat{\mathbf{s}}_{\infty}$, the primary stable (contracting) direction. Both the coordinate contraction and differential growth contribute to an exponential decay in $l_{m}$, so a uniform initial field would still develop exponentially finer structure in space unless $\lambda_{l}$ is independent of position, an event of little practical relevance. The exponential rate at which $l_{m}$ decays provides final information in determining the three dimensional spatial structures of the field distribution. Two factors require consideration. The magnitude of $\lambda_{m}$ in comparison with $\lambda_{s}$ and whether $\hat{\mathbf{m}}_{\infty} \cdot \nabla_{0} \lambda_{l} t$ has an exponential time dependence. Similar as the conjecture made earlier, we expect that $\lambda_{l}$ vary smoothly along the second stable direction $\hat{\mathbf{m}}_{\infty}$. Then the only deciding factor is the relative magnitude of $\lambda_{s}$ and $\lambda_{m}$. Unless $\lambda_{s}=\lambda_{m}$, in which case $l_{s} \approx l_{m} \propto \exp \left(\lambda_{s} t\right)$ so fields concentrate in tubes, the strong field appears in ribbons since $l_{s} \propto \exp \left(\lambda_{s} t\right) \ll l_{m} \propto \exp \left(\lambda_{m} t\right)$ for large $t$. These conclusions 
utilize the fact that $l_{e}$ varies at most algebraically in time. It should also be noted that these results come from the differential growth mechanism, and are independent whether the initial field has a spatial dependence or not.

In a large class of flows such as the three dimensional time-independent flows, $\lambda_{m}^{\infty}$ strictly vanishes. Hence $\lambda_{m}^{\infty}=0$ is a case of special interest. The characteristic direction $\hat{\mathbf{m}}_{\infty}$ associated with $\lambda_{m}^{\infty}=0$ is usually called the center (manifold) direction. It is a direction along which the separation between neighboring trajectories varies at most algebraically in time, which means $\Lambda_{m}$ is an algebraic function in $t$ if it has any time dependence at all. The time dependence of $\hat{\mathbf{m}}_{\infty} \cdot \nabla_{0}\left(\lambda_{l} t\right)$ is difficult to generalize. Numerical calculation in some cases has shown smooth variation of $\lambda_{l}$ along $\hat{\mathbf{m}}_{\infty}$ direction over large distance but there are intermittent wild fluctuations. The intermittence can be real, but numerical round-off can not be ruled out at this stage due to the delicacy of the calculation. There are three main scenarios. If $\hat{\mathbf{m}}_{\infty} \cdot \nabla_{0}\left(\lambda_{l} t\right)$ varies algebraically in time, $l_{m}$ is comparable with $l_{e}$ and the magnetic field accumulates in sheets. If $\hat{\mathbf{m}}_{\infty} \cdot \nabla_{0}\left(\lambda_{l} t\right) \propto \exp \left(\lambda_{l} t\right)$ the flux concentrates in tubes since $l_{m}$ is now comparable with $l_{s}$ due to $\lambda_{s} \approx-\lambda_{l}$ in a flow where $\nabla \cdot \mathrm{v}$ does not vary exponentially. Otherwise, the field has a ribbon like concentration. It requires a dominant contribution from the differential growth mechanism to achieve a tube or ribbon-like spatial distribution if $\lambda_{m}^{\infty}=0$.

Although the characteristic scale of the magnetic field along the $\mathcal{M}$ direction decides whether the field structure appears in tubes, ribbons, or sheets. It is the rich structure of the magnetic field in the $\mathcal{S}$ direction that inevitably leads the magnetic field to have a fractal-like spiky distribution in a chaotic dynamo [22]. The physical mechanisms responsible for the fine scales are the coordinate contraction that brings different initial fields close, and differential growth that comes from the spatial variation of local Lyapunov exponent in a general flow. The coordinate contraction mechanism is absent without an initial spatial gradient of the seed field. But differential growth always presents for a smooth flow. The only reason that the differential growth mechanism does not dominate the coordinate contraction mechanism in generating small scale structures is because the finite time Lyapunov exponent $\lambda_{l}$ varies 
smoothly along the stable (contracting) direction $\left(\hat{\mathbf{s}}_{\infty}\right)$, in contrast to the wild variation along the $\mathcal{E}$ direction. The ever finer field concentration assures the importance of a small but finite resistivity.

\section{NONIDEAL EVOLUTION OF A MAGNETIC FIELD: 2D CASE}

The competition between field growth and Ohmic dissipation has a clear resolution if the system is restricted to two dimensions. Although the lack of time-asymptotic dynamo action in two dimensions has a simple explanation, the characteristics of the solution were not known from previous anti-dynamo theorems. In particular, the nature of resistive dissipation was not known due to the lack of a full solution.

In this section we give a solution to the evolution of a two dimensional magnetic field in a chaotic flow and illustrate the time scale and magnitude of the Ohmic dissipation. We assume the initial magnetic energy is much smaller than the kinetic energy (specific requirements are discussed in section VI), so the back reaction of the magnetic field on the flow field can be neglected.

A two dimensional magnetic field can be represented by a scalar potential $\psi$, i.e., $\mathbf{B}(x, y, t)=\hat{\mathbf{z}} \times \nabla \psi(x, y, t)$. Substituting this relation into the induction equation, one obtains the advection-diffusion equation for $\psi(x, y, t)$ which describes the magnetic field evolution in two dimensions,

$$
\partial \psi / \partial t+\mathbf{v} \cdot \nabla \psi=\left(\eta / \mu_{0}\right) \nabla^{2} \psi
$$

The induced current density is given by the curl of the magnetic field

$$
\mathbf{j}=\nabla \times \mathbf{B}=-\nabla^{2} \psi \hat{\mathbf{z}}
$$

which strictly points in the $\hat{\mathbf{z}}$ direction. Hence $\mathbf{j} \cdot \mathbf{B}=0$ for two dimensional magnetic evolution, which is clearly different from the general three dimensional case as discussed in the previous section. In a chaotic plasma flow, one finds that

$$
B^{2} \approx B_{0}^{2} \exp (2 \lambda t) \text { and } \mu_{0}^{2} j^{2} \approx\left(B_{0} / L\right)^{2} \exp (4 \lambda t)
$$


and $\mathbf{j} \cdot \mathbf{B}=0, \mathbf{j} \times \mathbf{B} \propto \exp (3 \lambda t)$ before the resistivity becomes important.

As shown in [14], the advection-diffusion equation is an ordinary diffusion equation with a tensor diffusivity $D^{i j} \equiv\left(\eta / \mu_{0}\right) g^{i j}$ in Lagrangian coordinates,

$$
\frac{\partial \psi}{\partial t}=\frac{\partial}{\partial \xi^{i}} D^{i j} \frac{\partial}{\partial \xi^{j}} \psi
$$

For a two dimensional divergence-free flow as discussed here,

$$
D^{i j}=\left(\eta / \Lambda \mu_{0}\right) \hat{\mathbf{e}} \hat{\mathbf{e}}+\left(\eta \Lambda / \mu_{0}\right) \hat{\mathbf{s}} \hat{\mathbf{s}}
$$

with $\Lambda=\exp 2 \lambda t>1$. The diffusion equation is simplified in a natural Lagrangian coordinate system $(\alpha-\beta)$ which can be defined as

$$
\hat{\mathbf{e}}_{\infty}=a \nabla \alpha, \hat{\mathbf{s}}_{\infty}=b \nabla \beta, \text { and } \hat{\mathbf{e}}_{\infty}=\hat{\mathbf{z}} \times \hat{\mathbf{s}}_{\infty} .
$$

The Jacobian of the $\alpha-\beta$ coordinates is $a b$.

In natural Lagrangian coordinates $\alpha-\beta$, the diffusion equation is [14]

$$
\frac{\partial \psi}{\partial t}=-\frac{1}{a b} \frac{\partial}{\partial \alpha}\left(a b \gamma^{\alpha}\right)-\frac{1}{a b} \frac{\partial}{\partial \beta}\left(a b \gamma^{\beta}\right)
$$

where $\gamma^{\alpha}$ and $\gamma^{\beta}$ are the fluxes in the $\alpha$ and $\beta$ directions,

$$
\begin{aligned}
\gamma^{\alpha} & =-\frac{D_{e e}}{a^{2}} \frac{\partial \psi}{\partial \alpha}-\frac{D_{e s}}{a b} \frac{\partial \psi}{\partial \beta} \\
\gamma^{\beta} & =-\frac{D_{e s}}{a b} \frac{\partial \psi}{\partial \alpha}-\frac{D_{s s}}{b^{2}} \frac{\partial \psi}{\partial \beta}
\end{aligned}
$$

The diffusion coefficients are $D_{e e} \equiv \hat{\mathbf{e}}_{\infty} \cdot \overleftrightarrow{\mathbf{D}} \cdot \hat{\mathbf{e}}_{\infty}, D_{s e} \equiv \hat{\mathbf{s}}_{\infty} \cdot \overleftrightarrow{\mathbf{D}} \cdot \hat{\mathbf{e}}_{\infty}$, and $D_{s s} \equiv \hat{\mathbf{s}}_{\infty} \cdot \overleftrightarrow{\mathbf{D}} \cdot \hat{\mathbf{s}}_{\infty}$. For $\Lambda \gg 1$, these coefficients satisfy the inequalities $D_{e e} \approx\left(\eta / \mu_{0}\right) e^{-2 \lambda t} \ll D_{s e} \approx-\sigma\left(\eta / \mu_{0}\right) \ll$ $D_{s s} \approx\left(\eta / \mu_{0}\right) e^{2 \lambda t}$. The evolution of a two dimensional magnetic field can be illustrated by making the rough approximations that $a=b=1, \lambda$ a constant, and $\sigma=0[\sigma$ is defined in equation (5) and it measures the convergence rate of the eigenvectors]. Then an initial magnetic flux function $\psi(t=0)=c_{0}(1-\cos k \alpha)(1-\cos k \beta)$ relaxes as

$$
\psi=c_{0}\left\{1-\exp \left[-\left(1-e^{-2 \lambda t}\right) / 2 \Omega\right] \cos k \alpha\right\}\left\{1-\exp \left[-\left(e^{2 \lambda t}-1\right) / 2 \Omega\right] \cos k \beta\right\}
$$


with $\Omega \equiv \mu_{0} \lambda / k^{2} \eta$ since $1 / k$ is the characteristic length scale. The magnetic field is then given by

$$
B^{2}=\nabla_{0} \psi \cdot \stackrel{\leftrightarrow}{D} \cdot \nabla_{0} \psi=(\partial \psi / \partial \alpha)^{2} \exp (-2 \lambda t)+(\partial \psi / \partial \beta)^{2} \exp (2 \lambda t)
$$

with

$$
\begin{aligned}
& \frac{\partial \psi}{\partial \alpha}=c_{0}\left\{\exp \left[-\left(1-e^{-2 \lambda t}\right) / 2 \Omega\right] k \sin k \alpha\right\}\left\{1-\exp \left[-\left(e^{2 \lambda t}-1\right) / 2 \Omega\right] \cos k \beta\right\} \\
& \frac{\partial \psi}{\partial \beta}=c_{0}\left\{1-\exp \left[-\left(1-e^{-2 \lambda t}\right) / 2 \Omega\right] \cos k \alpha\right\}\left\{\exp \left[-\left(e^{2 \lambda t}-1\right) / 2 \Omega\right] k \sin k \beta\right\}
\end{aligned}
$$

The two terms in $B^{2}$ have completely different time dependence,

$$
\begin{aligned}
& \left(\frac{\partial \psi}{\partial \alpha}\right)^{2} e^{-2 \lambda t}=c_{0}^{2} k^{2} \sin ^{2} k \alpha\left\{1-\exp \left[-\left(e^{2 \lambda t}-1\right) / 2 \Omega\right] \cos k \beta\right\}^{2} \exp \left[-\frac{1-\exp (-2 \lambda t)}{\Omega}-2 \lambda t\right] \\
& \left(\frac{\partial \psi}{\partial \beta}\right)^{2} e^{2 \lambda t}=c_{0}^{2} k^{2} \sin ^{2} k \beta\left\{1-\exp \left[-\left(1-e^{-2 \lambda t}\right) / 2 \Omega\right] \cos k \alpha\right\}^{2} \exp \left[-\frac{\exp (2 \lambda t)-1}{\Omega}+2 \lambda t\right] .
\end{aligned}
$$

For $t<t_{a}-1 / \lambda$ with $t_{a} \equiv(\ln 2 \Omega) / 2 \lambda$ and $\Omega \equiv \mu_{0} \lambda L^{2} / \eta$,

$$
B^{2} \approx\left(\mathbf{B}_{0} \cdot \hat{\mathbf{e}}_{\infty}\right)^{2} \exp 2 \lambda t
$$

The magnetic field is dissipated away for a relatively short period $1 / \lambda$ centered on $t_{a}$. This is due to the Ohmic dissipation of the induced current,

$$
\begin{aligned}
\mu_{0}^{2} j^{2} & =\left[\frac{\partial^{2} \psi}{\partial \alpha \partial \beta}\left(e^{2 \lambda t}-e^{-2 \lambda t}\right)^{2}\right]^{2} \\
& =c_{0}^{2} k^{4} \sin ^{2} k \alpha \sin ^{2} k \beta h^{2}(t) \exp [-2 h(t) / \Omega],
\end{aligned}
$$

with

$$
h(t)=\exp (2 \lambda t)-\exp (-2 \lambda t)
$$

Since $B \propto \exp \lambda t, j \propto \exp 2 \lambda t$, and $\mathbf{j} \cdot \mathbf{B}=0$, the Lorentz force on the flow grows exponentially in time with $|\mathbf{j} \times \mathbf{B}| \propto \exp 3 \lambda t$.

The production of heat by the Ohmic dissipation, $\eta j^{2}$, is concentrated in a relatively short time interval $1 / \lambda$, which coincides with the diminution of the main magnetic energy. The total energy dissipated is larger by a factor of $\Omega \equiv \lambda L^{2} \mu_{0} / \eta$ than the initial magnetic energy $\int\left(B_{0}^{2} / 2 \mu_{0}\right) d^{3} \mathbf{x}$. The additional energy comes from the flow during the advection period $t_{a}$ in 
which the field is frozen to the flow. In actuality, the finite time Lyapunov exponent is not a constant and an energetic plasma flow tends to generate complicated dissipative structures given a small seed magnetic field. The spatial-temporal complexity of the Ohmic dissipation is determined by the spatial-temporal dependence of the finite time Lyapunov exponent.

\section{RESISTIVE DISSIPATION: FILAMENTS AND SHEETS}

In section III, it was shown that the ideal evolution of a magnetic field in a chaotic plasma leads to an exponentially growing magnetic field and induced current density. This suggests that a finite resistivity, regardless of how small it might be, will be important and changes the characteristics of the solution. In general, the magnetic field initially evolves ideally

and then dissipation dominates the solution if the plasma flow is chaotic. The heating, $\eta j^{2}$, is concentrated in the current channels at the end of an ideal evolution. The cut-off time for ideal evolution is approximated by $\tau_{c}=(\ln 2 \Omega) / 2|\lambda|$ with $\Omega \equiv \mu_{0}|\lambda| L^{2} / \eta$. Since the dimensionless number $\Omega$ is usually large $\left(>10^{8}\right)$, the magnetic field, which follows an ideal evolution before $t_{a}$, would have obtained extreme spatial anisotropy and fine scales at the onset of efficient Ohmic dissipation $t_{a}$. For the same reason, the spatial distribution of the Ohmic dissipation can be learned by following the ideal evolution of the induced current density, which is given by the curl of the magnetic field.

In the case of a two dimensional magnetic field, the production of heat by the Ohmic dissipation, $\eta j^{2}$, is concentrated in a relatively short time interval $1 / \lambda$, which coincides with the dissipation of the main magnetic energy. The total energy dissipated is larger by a factor of $\Omega \equiv \lambda L^{2} \mu_{0} / \eta$ than the initial magnetic energy $\int\left(B_{0}^{2} / 2 \mu_{0}\right) d^{3} \mathbf{x}$. The additional energy comes from the flow during the advection period $\tau_{c}$ in which the field is frozen to the flow.

In the general three dimensional case, the current tends to flow along the $\mathcal{E}$ direction, just like the magnetic field. To see this, we note that the current density has the form

$$
\mathbf{j}=\sum j^{i}\left(\partial \mathbf{x} / \partial \xi^{i}\right)
$$


with

$$
\mu_{0} j^{i}=\sum_{j k l} \frac{\epsilon^{i j k}}{J} \frac{\partial}{\partial \xi^{j}}\left[g_{k l} \frac{b^{l}(\xi)}{J}\right] .
$$

The $\mathcal{E}$ component of the current density is

$$
j_{e} \equiv \mathbf{j} \cdot \mathcal{E} /|\mathcal{E}|=\mu_{0}^{-1} \Lambda_{l}^{3 / 2}\left(\hat{\mathbf{e}}_{\infty} \cdot \mathbf{B}_{0}\right) \hat{\mathbf{e}}_{\infty} \cdot \nabla_{0} \times \hat{\mathbf{e}}_{\infty}+O\left(\Lambda_{l}\right)
$$

The $\mathcal{S}$ component of the current density is the smallest,

$j_{s} \equiv \mathbf{j} \cdot \mathcal{S} /|\mathcal{S}|=\mu_{0}^{-1} \sqrt{\Lambda_{s}} \Lambda_{l}\left[\left(\hat{\mathbf{e}}_{\infty} \cdot \mathbf{B}_{0}\right) \hat{\mathbf{s}}_{\infty} \cdot \nabla_{0} \times \hat{\mathbf{e}}_{\infty}-\hat{\mathbf{m}}_{\infty} \cdot \nabla_{0}\left(\hat{\mathbf{e}}_{\infty} \cdot \mathbf{B}_{0}\right)-\left(\hat{\mathbf{e}}_{\infty} \cdot \mathbf{B}_{0}\right) \hat{\mathbf{m}}_{\infty} \cdot \nabla_{0}\left(2 \lambda_{l} t\right)\right]+O\left(\Lambda_{s}\right)$,

where we have used the convention $\hat{\mathbf{e}} \times \hat{\mathbf{m}}=\hat{\mathbf{s}}$. The $\mathcal{M}$ component of the current density is

$j_{m} \equiv \mathbf{j} \cdot \mathcal{M} /|\mathcal{M}|=\mu_{0}^{-1} \sqrt{\Lambda_{m}} \Lambda_{l}\left(\hat{\mathbf{e}}_{\infty} \cdot \mathbf{B}_{0}\right)\left[\hat{\mathbf{m}}_{\infty} \cdot \nabla_{0} \times \hat{\mathbf{e}}_{\infty}+\hat{\mathbf{s}}_{\infty} \cdot \nabla_{0} \ln \left(\hat{\mathbf{e}}_{\infty} \cdot \mathbf{B}_{0}\right)+\hat{\mathbf{s}}_{\infty} \cdot \nabla_{0}\left(2 \lambda_{l} t\right)\right]+O\left(\Lambda_{m}\right)$.

In the case that $\hat{\mathbf{m}}_{\infty} \cdot \nabla_{0}\left(2 \lambda_{l} t\right)$ does not have an exponential time dependence, the current density obeys

$$
j_{e} \propto e^{3 \lambda_{l} t} \gg j_{m} \propto e^{2 \lambda_{l} t+\lambda_{m} t} \gg j_{s} \propto e^{2 \lambda_{l} t+\lambda_{s} t} .
$$

In a time-independent flow, $\lambda_{l} \approx-\lambda_{s} \approx \lambda>0$ and $\lambda_{m} \approx 0$, one has

$$
j_{e} \propto e^{3 \lambda t} \gg j_{m} \propto e^{2 \lambda t} \gg j_{s} \propto e^{\lambda t}
$$

If $\hat{\mathbf{m}}_{\infty} \cdot \nabla_{0}\left(\lambda_{l} t\right) \propto e^{\lambda_{l} t}$, one finds instead

$$
j_{e} \propto e^{3 \lambda t} \gg j_{m} \approx j_{s} \propto e^{2 \lambda t}
$$

In either case, the natural state is for the induced current to align with the magnetic field.

The spatial scale of the current density distribution has strong anisotropy similar as the magnetic field. To see this, we calculate the spatial scale of the current density distribution along different characteristic directions. We consider the general case that the flow is truly three dimensional which means $\hat{\mathbf{e}}_{\infty} \cdot \nabla_{0} \times \hat{\mathbf{e}}_{\infty}$ does not vanish. In this case, the leading order contribution to $j^{2}$ is $\Lambda_{l}^{3}\left(\hat{\mathbf{e}}_{\infty} \cdot \mathbf{B}_{0}\right)^{2}\left(\hat{\mathbf{e}}_{\infty} \cdot \nabla_{0} \times \hat{\mathbf{e}}_{\infty}\right)^{2}$. The gradient of $j^{2}$ in the $\mathcal{E}$ direction is

$$
\frac{\mathcal{E} \cdot \nabla j^{2}}{|\mathcal{E}| j^{2}}=\frac{6 \hat{\mathbf{e}}_{\infty} \cdot \nabla\left(\lambda_{l} t\right)}{\sqrt{\Lambda_{l}}}+O\left(e^{-\lambda_{l} t}\right) .
$$


The spatial scale along the $\mathcal{E}$ direction always has an algebraic dependence in time because of $\hat{\mathbf{e}}_{\infty} \cdot \nabla_{0} \lambda_{l} t \propto \exp \left(\lambda_{l} t\right)$

$$
l_{e} \equiv\left[\frac{\mathcal{E} \cdot \nabla j^{2}}{|\mathcal{E}| j^{2}}\right]^{-1} \approx \frac{\sqrt{\Lambda_{l}}}{6 \hat{\mathbf{e}}_{\infty} \cdot \nabla\left(\lambda_{l} t\right)} \approx l_{e}^{0} t^{q} .
$$

Just like the magnetic field, the spatial scale of the current density distribution along the $\mathcal{S}$ direction tends to diminish at an exponential rate $\left(\lambda_{s}<0\right)$,

$$
l_{s} \equiv\left[\frac{\mathcal{S} \cdot \nabla j^{2}}{|\mathcal{S}| j^{2}}\right]^{-1} \approx l_{s}^{0} \exp \left(\lambda_{s} t\right)
$$

with $l_{s}^{0}$ determined by both the flow field and the initial magnetic field configuration,

$$
\begin{aligned}
l_{s}^{0}= & \left\{6 \hat{\mathbf{s}}_{\infty} \cdot \nabla_{0}\left(\lambda_{l} t\right)+\hat{\mathbf{s}}_{\infty} \cdot \nabla_{0} \ln \left(\mathbf{B}_{0} \cdot \hat{\mathbf{e}}_{\infty}\right)^{2}\right. \\
& \left.+\hat{\mathbf{s}}_{\infty} \cdot \nabla_{0} \ln \left(\hat{\mathbf{e}}_{\infty} \cdot \nabla_{0} \times \hat{\mathbf{e}}_{\infty}\right)^{2}\right\}^{-1} .
\end{aligned}
$$

The spatial scale along the $\mathcal{M}$ direction, in leading order, is

$$
\begin{aligned}
l_{m} \equiv & {\left[\frac{\mathcal{M} \cdot \nabla j^{2}}{|\mathcal{M}| j^{2}}\right]^{-1} } \\
\approx & \left\{6\left(\hat{\mathbf{m}}_{\infty} \cdot \nabla_{0} \lambda_{l} t\right)+\hat{\mathbf{m}}_{\infty} \cdot \nabla_{0} \ln \left(\mathbf{B}_{0} \cdot \hat{\mathbf{e}}_{\infty}\right)^{2}\right. \\
& \left.+\hat{\mathbf{m}}_{\infty} \cdot \nabla_{0} \ln \left(\hat{\mathbf{e}}_{\infty} \cdot \nabla_{0} \times \hat{\mathbf{e}}_{\infty}\right)^{2}\right\}^{-1} \sqrt{\Lambda_{m}}
\end{aligned}
$$

If the middle Lyapunov exponent is negative, $l_{m}$ is exponentially reduced similar as $l_{s}$ so the current concentrates in elongated tubes or ribbon-like structures depending on the ratio between $\lambda_{m}$ and $\lambda_{s}$. When the middle Lyapunov exponent is positive, one expects that $\hat{\mathbf{m}}_{\infty} \cdot \nabla_{0}\left(\lambda_{l} t\right)$ has an exponential time dependence so current distribution will not be smoothed out indefinitely. In this case, the asymptotic form for $l_{m}$ is given by

$$
l_{m} \approx \frac{\exp \left(\lambda_{m} t\right)}{\hat{\mathbf{m}}_{\infty} \cdot \nabla_{0}\left(6 \lambda_{l} t\right)}
$$

In the case that $\lambda_{m}^{\infty}$ vanishes, which is always true for a time-independent flow, $\Lambda_{m}$ varies at most algebraically in time. $l_{m}$ varies algebraically in time if $\hat{\mathbf{m}}_{\infty} \cdot \nabla_{0} \lambda_{l} t$ does not blow up exponentially in time. In that case, $l_{s} \ll l_{m} \approx l_{e}$, hence the Ohmic heating occurs in current 
sheets. On the other hand, if $\hat{\mathbf{m}}_{\infty} \cdot \nabla_{0} \lambda_{l}$ t grows exponentially in time, one finds $l_{s} \approx l_{m} \ll l_{e}$ and the Ohmic heating occurs in current filaments.

In general, the differential growth $\nabla_{0}\left(\lambda_{l} t\right)$ plays a dominant role in determining the characteristic scales of the current (and magnetic field) distribution along the $\hat{\mathbf{m}}_{\infty}$ and $\hat{\mathbf{e}}_{\infty}$ directions, in comparison with the other factors such as coordinate contraction, which operate only if the initial seed field has a spatial dependence. The current runs through tube, sheet, or ribbon-like structures if conditions on $\left(\lambda_{l}, \lambda_{m}, \lambda_{s}\right)$ and $\nabla_{0}\left(\lambda_{l} t\right)$ are met in a particular flow. The analysis of $\left(\lambda_{l}, \lambda_{m}, \lambda_{s}\right)$ and $\nabla_{0}\left(\lambda_{l} t\right)$ is straightforward once the flow field is specified. As a general result, we have found that $\nabla_{0}\left(\lambda_{l} t\right)$ is well-behaved in the $\hat{\mathbf{s}}_{\infty}$ direction but ill-behaved in the $\hat{\mathbf{e}}_{\infty}$ direction (i.e. $\hat{\mathbf{e}}_{\infty} \cdot \nabla_{0}\left(\lambda_{l} t\right)$ exponentiates in time). The $\hat{\mathbf{m}}_{\infty} \cdot \nabla_{0}\left(\lambda_{l} t\right)$ requires a case-by-case examination.

It should be stressed that the onset of Ohmic heating has a large spread in time due to the spatial variation of the local Lyapunov exponents. To understand the distribution of Ohmic heating power as a function of time, one can convolute the time $\tau_{c}$ with the proper probability distribution function of the finite time Lyapunov exponents for any given flow under consideration.

The difference between the distribution of the finite time Lyapunov exponents and a Gaussian distribution becomes smaller as one samples the finite time Lyapunov exponent at a longer interval (larger $t_{0}$ in $\lambda\left(\xi, t_{0}\right)$ ). For large $t_{0}$ (compared with the Lyapunov time), the standard deviation of the distribution of the finite time Lyapunov exponents scales as $1 / \sqrt{t_{0}}$. This implies that if the plasma resistivity is smaller and the flow is more chaotic, the histogram of the heating is closer to Gaussian statistics and the spread in time for the main Ohmic heating is smaller.

Locations with peculiarly small finite time Lyapunov exponents are barriers for the Ohmic dissipation. These regions are where the $\hat{\mathbf{s}}$ lines make sharp bends (see reference [14] for the two dimensional case, the three dimensional case is discussed in reference [15], additional numerical evidence is also shown in reference [23]). The existence of transport barriers in highly chaotic plasma flows implies the strong intermittence of the Ohmic dis- 
sipation of a magnetic field in a chaotic conducting fluid. The parts of the chaotic region with flat $\hat{\mathbf{s}}$ lines will appear to be hot and dissipate most of the energy at the onset of the rapid Ohmic dissipation due to the entangling of magnetic field lines, while the transport barriers (regions with large $\hat{\mathbf{s}}$ line curvature) have low dissipation and remain dark. On a longer time scale, the transport barrier is the location of strong dissipation and becomes bright while the vast chaotic region is dark. This brightness can last significantly longer than the initial main burst of Ohmic dissipation in the chaotic region. A transport barrier in the chaotic region can be easily distinguished from the regular Kolmogorov-Arnold-Moser (KAM) islands since the Ohmic dissipation in the regular islands is on a much longer time scale. The regular islands are always dark on the time scale in which the transport barrier in a chaotic region lives and dies, i.e. from dark to bright and then back to dark again.

\section{BACK REACTION OF THE MAGNETIC FIELD ON THE FLOW}

To solve the magnetic field evolution problem self-consistently, one has to consider the back reaction of the magnetic field on the plasma flow field [9]. There are a number of unique features of this back reaction if the flow is chaotic. We will first examine the two dimensional case which admits a clear long time solution to the non-ideal magnetic field evolution just like the two dimensional passive scalar problem (section IV or reference [14]). Although a similar solution to the non-ideal evolution of a general three dimensional magnetic field in a chaotic flow is not available, the back reaction on the flow has generic features which can be related to the spatial and temporal dependence of the finite time Lyapunov exponents.

There are essentially two issues. The first is whether the back reaction on the flow field is important before the Ohmic dissipation removes the magnetic energy (in two dimensions) or suppress the transient growth of the magnetic field (as could happen in three dimensions). If the back reaction does become important before dissipation, then the second issue is to resolve the effects of the Lorentz force on the flow field.

The magnetic field affects the flow field through the Lorentz force $\mathbf{j} \times \mathbf{B}$ on the plasma. 
In the two dimensional case, $\mathbf{B}=\hat{\mathbf{z}} \times \nabla \psi(x, y, t)$ and $\mathbf{j}=-\nabla^{2} \psi \hat{\mathbf{z}}$. Hence the magnitude of the Lorentz force is the product of the magnitudes of the current density and the magnetic field,

$$
|\mathbf{j} \times \mathbf{B}|=j B \approx\left(B_{0}^{2} / L\right) \exp (3 \lambda t)
$$

This force should be compared with the convective force $\rho v^{2} / L$, where $\rho$ is the density of the media and $L$ is the characteristic scale of the flow. The back reaction of the magnetic field on the plasma flow is negligible if the Lorentz force is much smaller than the convective force which drives the initial flow field. This is satisfied for time $t<t_{b}$ with

$$
t_{b} \equiv(1 / 3 \lambda) \ln \left(\epsilon^{k} / \epsilon_{0}^{m}\right)
$$

where $\epsilon^{k}$ is the kinetic energy density $\rho v^{2} / 2$ and $\epsilon_{0}^{m}$ is the initial magnetic energy density $B_{0}^{2} / 2 \mu_{0}$. The back reaction of the magnetic field on the plasma flow with finite resistivity is negligible if $t_{b}>\tau_{c} \equiv(\ln 2 \Omega) / 2 \lambda$ with $\Omega \equiv \mu_{0} \lambda l^{2} / \eta$, i.e.,

$$
\Omega<\left(\epsilon^{k} / \epsilon_{0}^{m}\right)^{2 / 3}
$$

If $\Omega>\left(\epsilon^{k} / \epsilon_{0}^{m}\right)^{2 / 3}$, the effect of the back reaction of the magnetic field on the plasma flow will be important, and the flow field will be modified before the Ohmic dissipation removes the magnetic energy. For $t<\tau_{c}$, the exponentially growing magnetic field lies along the $\hat{\mathbf{E}}$ direction of the flow, $B_{e} \approx\left(\mathbf{B}_{0} \cdot \hat{\mathbf{e}}_{\infty}\right) \exp (\lambda t) \gg B_{s} \approx\left(\mathbf{B}_{0} \cdot \hat{\mathbf{s}}_{\infty}\right) \exp (-\lambda t)$. Since the current density strictly lies along the $\hat{\mathbf{z}}$ direction, $\mathbf{j}=-\nabla^{2} \psi \hat{\mathbf{z}}$, the Lorentz force points at the $\mathcal{S}$ direction. Since $\nabla^{2} \psi \propto \exp (2 \lambda t)$, the $\mathcal{S}$ component of the Lorentz force is

$$
(\mathbf{j} \times \mathbf{B}) \cdot \mathcal{S} /|\mathcal{S}|=B_{e} \nabla^{2} \psi \propto \exp (3 \lambda t) .
$$

The $\mathcal{E}$ component of the Lorentz force is much smaller with

$$
(\mathbf{j} \times \mathbf{B}) \cdot \mathcal{E} /|\mathcal{E}|=-B_{s} \nabla^{2} \psi \propto \exp (\lambda t) .
$$

Since

$$
\frac{[(\mathbf{j} \times \mathbf{B}) \cdot \mathcal{S} /|\mathcal{S}|]}{[\mathbf{j} \times \mathbf{B} \cdot \mathcal{E} /|\mathcal{E}|]} \propto \exp (2 \lambda t)
$$


the Lorentz force primarily applies along the $\mathcal{S}$ line of the flow.

The primary difference between the two dimensional case and the generic three dimensional magnetic field evolution is the alignment of the induced current density and the magnetic field in three dimensions. It was shown in section III that the magnetic field $\mathbf{B}=\sum b^{i}\left(\partial \mathbf{x} / \partial \xi^{i}\right)$ is along the $\mathcal{E}$ direction, $B_{\epsilon} \equiv \mathbf{B} \cdot \mathcal{E} /|\mathcal{E}| \approx\left(\mathbf{B}_{0} \cdot \hat{\mathbf{e}}_{\infty}\right) \exp \lambda t \gg B_{m} \equiv$ $\mathbf{B} \cdot \mathcal{M} /|\mathcal{M}| \approx \mathbf{B}_{0} \cdot \hat{\mathbf{m}}_{\infty} \gg B_{s} \equiv \mathbf{B} \cdot \hat{\mathbf{S}} /|\hat{\mathbf{S}}|=\left(\mathbf{B}_{0} \cdot \hat{\mathbf{s}}_{\infty}\right) \exp (-\lambda t)$. The current density $\mathbf{j}=\sum j^{i}\left(\partial \mathbf{x} / \partial \xi^{i}\right)$ with

$$
\mu_{0} j^{i}=\sum_{j k l}\left(\epsilon^{i j k} / J\right) \partial / \partial \xi^{j}\left[g_{k l} b^{l}(\xi) / J\right]
$$

also lies along the $\mathcal{E}$ direction, as shown in section III. As a consequence of the alignment between $\mathbf{B}$ and $\mathbf{j}$, the Lorentz force still scales $\exp \left(3 \lambda_{l} t\right)$, just as the two dimensional case, despite $B \propto e^{\lambda_{l} t}$ and $j \propto e^{3 \lambda_{l} t}$.

The spatial breakdown of the Lorentz force can be illustrated with the class of timeindependent flows. In such flows, the orientation of the Lorentz force depends on the spatial variation of the finite time Lyapunov exponent along the $\hat{\mathbf{m}}_{\infty}$ direction. If the finite time Lyapunov exponent has a gradient along the $\mathcal{M}$ direction which varies algebraically in time, the Lorentz force points at the $\mathcal{S}$ direction with

$$
\begin{aligned}
f_{s} & \equiv(\mathbf{j} \times \mathbf{B}) \cdot \mathcal{S} /|\mathcal{S}|=j_{e} B_{m}-j_{m} B_{e} \propto \exp (3 \lambda t), \\
f_{m} & \equiv(\mathbf{j} \times \mathbf{B}) \cdot \mathcal{M} /|\mathcal{M}|=j_{s} B_{e}-j_{e} B_{s} \propto \exp (2 \lambda t), \\
f_{e} & \equiv(\mathbf{j} \times \mathbf{B}) \cdot \mathcal{E} /|\mathcal{E}|=j_{m} B_{s}-j_{s} B_{m} \propto \exp (\lambda t) .
\end{aligned}
$$

If the finite time Lyapunov exponent develops an exponentially increasing gradient along the $\hat{\mathbf{m}}_{\infty}$ direction, one finds $f_{s} \approx f_{m} \propto \exp (3 \lambda t) \gg f_{e} \propto \exp \lambda t$, i.e., the Lorentz force lies in the $(\mathcal{M}, \mathcal{S})$ plane. It is interesting to note that the magnetic field evolution in the first case leads to the formation of current sheets, while in the second case it generates current filaments. 


\section{SUMMARY}

The natural evolution of a magnetic field embedded in a chaotic conducting flow is to develop small scale structures with extreme spatial anisotropy, which typically take the form of filaments, ribbons, and sheets. For a turbulent background flow, the small scale structures of the magnetic field and induced current density may be attributed to the small scale structures of the turbulent flow velocity field itself. The same can not be said for nonintegrable smooth laminar flows. Instead the explanation is found in the kinematics of the chaotic Lagrangian trajectories of the flow. It is the spatial anisotropy of the finite time or local Lyapunov exponents along the various characteristic (un)stable directions, that causes the complicated structures for the magnetic field evolution.

The local Lyapunov exponent and the characteristic (un)stable directions are naturally defined as functions of Lagrangian coordinates and time, in the same spirit as chaos of flow trajectory means sensitive dependence on initial position (Lagrangian coordinates). The spatial dependence of the local Lyapunov exponent is described by two convergence functions $\tilde{\lambda}(\xi)$ and $f(\xi, t)$. The first one $\tilde{\lambda}(\xi)$ is entirely determined by the geometry of the stable foliation (the $\hat{\mathbf{s}}_{\infty}$ lines) and is a well-behaved smooth function of position alone. $f(\xi, t)$ is the one that has a strong spatial anisotropy. It does not vary along the $\hat{\mathbf{s}}_{\infty}$ direction, but develops exponentially increasing gradient (in time) along the most unstable direction $\hat{\mathbf{e}}_{\infty}$. The property of $f(\xi, t)$ along the $\hat{\mathbf{m}}_{\infty}$, the direction along which the middle Lyapunov exponent $\lambda_{m}$ (e.g. $\lambda_{m}^{\infty}=0$ for a time-independent flow) resides, is not unique. Whether $f(\xi, t)$ develops exponentially growing spatial gradient in this direction helps determine the exact form of the small scale structures, filaments, ribbons, or sheets.

We have identified three orthogonal directions $[\mathcal{S}, \mathcal{E}, \mathcal{M}$, as defined in equation (6)] in the Eulerian frame or real space that characterize the spatial anisotropy of the physical observable such as the magnetic field, induced current density, and the Lorentz force. For a chaotic plasma flow with a high magnetic Reynolds number, the initial stage of the magnetic field evolution is ideal and an initial small seed field grows exponentially in time, so does 
the induced current density except at an rate two times faster than the magnetic field. The induced current tends to align with the magnetic field, and both of them point along the $\mathcal{E}$ direction. This is also the direction along which the field and the current have the least structure. At the same time, both the magnetic field and the induced current density develop exponentially decreasing spatial scales in the $\mathcal{S}$ direction, which is transverse to the magnetic flux and current flow. The Lorentz force primarily acts along the $\mathcal{S}$ direction, which is transverse to the filament or sheets where magnetic flux and induced current concentrate. This is also the direction along with neighboring flow points converge exponentially in time. In other words, the Lorentz force primarily counter-acts the convective force (e.g. the pressure gradient) that squeezes neighboring flow points together. Consequently it suppresses Lagrangian chaos and regularizes the flow.

\section{ACKNOWLEDGMENTS}

This work was supported by U.S. Department of Energy.

\section{APPENDIX A: THE ASYMPTOTIC EXPRESSION FOR THE CURRENT DENSITY}

We will work with the divergence-free flow case so the Jacobian of the Lagrangian co-

ordinates is unity. The more general case has a similar expression. First we consider the expression

$$
\frac{\partial}{\partial \xi^{n}} \epsilon^{i n m} \frac{g_{m l}}{J} b^{l}
$$

which is equal to

$$
\frac{\partial}{\partial \xi^{n}}\left\{\epsilon^{i n m}\left[\Lambda_{l} \epsilon_{m}\left(\hat{\mathbf{e}} \cdot \mathbf{B}_{0}\right)+\Lambda_{m} m_{m}\left(\hat{\mathbf{m}} \cdot \mathbf{B}_{0}\right)+\Lambda_{s} s_{m}\left(\hat{\mathbf{s}} \cdot \mathbf{B}_{0}\right)\right]\right\}
$$

upon substituting the diagonal form of the metric tensor. In compact vector algebra form, the above expression is the $i$ th component of the curl

$$
\nabla_{0} \times\left[\Lambda_{l}\left(\hat{\mathbf{e}} \cdot \mathbf{B}_{0}\right) \hat{\mathbf{e}}+\Lambda_{m}\left(\hat{\mathbf{m}} \cdot \mathbf{B}_{0}\right) \hat{\mathbf{m}}+\Lambda_{s}\left(\hat{\mathbf{s}} \cdot \mathbf{B}_{0}\right) \hat{\mathbf{s}}\right]
$$


The full expression for $\mu_{0}^{2} j^{2}$ is then

$$
\begin{aligned}
\mu_{0}^{2} j^{2}= & \nabla_{0} \times\left[\Lambda_{l}\left(\hat{\mathbf{e}} \cdot \mathbf{B}_{0}\right) \hat{\mathbf{e}}+\Lambda_{m}\left(\hat{\mathbf{m}} \cdot \mathbf{B}_{0}\right) \hat{\mathbf{m}}+\Lambda_{s}\left(\hat{\mathbf{s}} \cdot \mathbf{B}_{0}\right) \hat{\mathbf{s}}\right] \\
& \cdot\left(\Lambda_{l} \hat{\mathbf{e}} \hat{\mathbf{e}}+\Lambda_{m} \hat{\mathbf{m}} \hat{\mathbf{m}}+\Lambda_{s} \hat{\mathbf{s}} \hat{\mathbf{s}}\right) \\
& \cdot \nabla_{0} \times\left[\Lambda_{l}\left(\hat{\mathbf{e}} \cdot \mathbf{B}_{0}\right) \hat{\mathbf{e}}+\Lambda_{m}\left(\hat{\mathbf{m}} \cdot \mathbf{B}_{0}\right) \hat{\mathbf{m}}+\Lambda_{s}\left(\hat{\mathbf{s}} \cdot \mathbf{B}_{0}\right) \hat{\mathbf{s}}\right] .
\end{aligned}
$$

The leading order term is

$$
\Lambda_{l}^{3}\left(\hat{\mathbf{e}} \cdot \mathbf{B}_{0}\right)^{2}(\hat{\mathbf{e}} \cdot \nabla \times \hat{\mathbf{e}})^{2}
$$

which has a time dependence of $\exp 6 \lambda_{l} t$ with $\lambda_{l}>0$ in a chaotic region of the flow. The leading order would identically vanish throughout space if $\hat{\mathbf{e}} \cdot \nabla_{0} \times \hat{\mathbf{e}}=0$ strictly holds. In the long time limit, that corresponds to $\hat{\mathbf{e}}_{\infty} \cdot \nabla_{0} \times \hat{\mathbf{e}}_{\infty}=0$.

The next order term has the form

$$
\Lambda_{m} \Lambda_{l}^{2}\left[\left(\hat{\mathbf{e}} \cdot \mathbf{B}_{0}\right) \hat{\mathbf{s}} \cdot \nabla_{0}\left(2 \lambda_{l} t\right)+\hat{\mathbf{s}} \cdot \nabla_{0}\left(\hat{\mathbf{e}} \cdot \mathbf{B}_{0}\right)+\left(\hat{\mathbf{e}} \cdot \mathbf{B}_{0}\right) \hat{\mathbf{m}} \cdot \nabla_{0} \times \hat{\mathbf{e}}\right]^{2},
$$

which has the time dependence of $\exp \left(4 \lambda_{l} t+2 \lambda_{m} t\right)$. In making this statement we have invoke the result that $\hat{\mathbf{s}}_{\infty} \cdot \nabla_{0}\left(\lambda_{l} t\right)$ is a function of position alone, or $\hat{\mathbf{s}} \cdot \nabla_{0}\left(\lambda_{l} t\right)$ has a very weak time dependence for large $t$.

There is one more term that might be of comparable magnitude if $\hat{\mathbf{m}} \cdot \nabla_{0}\left(\lambda_{l} t\right)$ has an exponential time dependence,

$$
\Lambda_{s} \Lambda_{l}^{2}\left[\hat{\mathbf{m}} \cdot \nabla_{0}\left(2 \lambda_{l} t\right)\right]^{2}\left(\hat{\mathbf{e}} \cdot \mathbf{B}_{0}\right)^{2}
$$

The hope is for $\Lambda_{s}\left[\hat{\mathbf{m}} \cdot \nabla_{0}\left(2 \lambda_{l} t\right)\right]^{2} \approx \Lambda_{m}$ which is obviously true if $\hat{\mathbf{m}} \cdot \nabla_{0}\left(2 \lambda_{l} t\right) \propto \exp \left(\lambda_{l} t\right)$. 


\section{REFERENCES}

[1] J.D. Jackson, Classical Electrodynamics Second Edition (John Wiley \& Sons, New York, 1975) Page 472.

[2] F. Krause and K.-H. Rädler, Mean-field magnetohydrodynamics and dynamo theory (Pergamon, Oxford, 1980).

[3] A.H. Boozer, J. Plasma Physics 35 (1986) 133.

[4] A. Bhattacharjee and Y. Yuan, Astrophys. J. 449 (1995) 739.

[5] A.V. Gruzinov and P.H. Diamond, Phys. Plasmas 3 (1996) 1853.

[6] M. Steenbeck, F. Krause, and K.-H. Rädler, Z. Naturforsch. 21a (1966) 369.

[7] H. Alfvén, Tellus 2 (1950) 74-82.

[8] V.I. Arnold, Ya. B. Zeldovich, A.A. Ruzmaikin, and D.D. Sokoloff, Sov. Phys. JETP 54 (1981) 1083-1086.

[9] S. Childress and A. D. Gilbert, Stretch, Twist, Fold: The Fast Dynamo (Springer, New York, 1995), and references therein.

[10] F. Cattaneo and S.I. Vainshtein, Ap. J. 376 L21 (1991); S.I. Vainshtein and F. Cattaneo, Ap. J. 393 (1992) 165.

[11] A. Pouquet, U. Frisch, and J. Leorat, J. Fluid Mech. 77 (1976) 321.

[12] E. Ott, Physics of Plasmas 5, (1998) 1636-1646, and references therein.

[13] E. Ott, Chaos in dynamical systems (Cambridge University Press, Cambridge, 1993).

[14] X.Z. Tang and A.H. Boozer, Physica D 95 (1996) 283-305.

[15] X.Z. Tang and A.H. Boozer, Physics of Fluids 6 (1999) 1418-1436.

[16] T..J. Taylor, Nonlinearity 63 (1993) 369-375. 
[17] S. Lundquist, Phys. Rev. 83 (1951) 307.

[18] A.H. Boozer, Astrophys. J. 394 (1992) 357-362.

[19] J.-P. Eckmann and D. Ruelle, Rev. Mod. Phys. 57 (1985) 617.

[20] V.I. Oseledec, Trans. Moscow Math. Soc. 19 (1968) 197-231.

[21] X.Z. Tang and A.H. Boozer, Phys. Lett. A 236 (1997) 476-482.

[22] S.I. Vainshtein, R.Z. Sagdeev, R. Rosner, Eun-Jin Kim, Phys. Rev. E 53 (1996) 4729-44.

[23] X.Z. Tang and A.H. Boozer, Chaos 9 (1999) 183-194. 